$\underline{\text { Preprint typeset in JHEP style. - HYPER VERSION }}$

IFUM-707-FT

hep-th/0202073

\title{
The Asymptotic Dynamics of two-dimensional (anti-)de Sitter Gravity
}

\author{
Mauro Brigante \\ Dipartimento di Fisica dell'Università di Milano \\ Via Celoria 16, I-20133 Milano. \\ E-mail: maubriga@tin.it
}

\section{Sergio Cacciatori}

Dipartimento di Matematica dell'Università di Milano

Via Saldini 50, I-20133 Milano and

INFN, Sezione di Milano, Via Celoria 16, I-20133 Milano.

E-mail: cacciatori@mi.infn.it

\section{Dietmar Klemm and Daniela Zanon \\ Dipartimento di Fisica dell'Università di Milano \\ Via Celoria 16, I-20133 Milano and \\ INFN, Sezione di Milano, Via Celoria 16, I-20133 Milano. \\ E-mail: dietmar.klemm@mi.infn.it, daniela.zanon@mi.infn.it}

\begin{abstract}
We show that the asymptotic dynamics of two-dimensional de Sitter or anti-de Sitter Jackiw-Teitelboim (JT) gravity is described by a generalized two-particle Calogero-Sutherland model. This correspondence is established by formulating the JT model of (A)dS gravity in two dimensions as a topological gauge theory, which reduces to a nonlinear 0+1-dimensional sigma model on the boundary of (A)dS space. The appearance of cyclic coordinates allows then a further reduction to the Calogero-Sutherland quantum mechanical model.
\end{abstract}

KEYwords: AdS-CFT Correspondence, 2D Gravity, Field Theories in Lower Dimensions. 


\section{Contents}

1. Introduction 1

2. Two-dimensional (anti-)de Sitter gravity as a topological gauge theory

3. Asymptotic behaviour of the fields 4

4. Boundary terms in the action 6

5. Further reduction 8

6. Positive cosmological constant 8

7. Conclusions 9

A. Conventions 11

A.1 Anti-de Sitter 11

A.2 De Sitter 11

B. $\mathrm{dS}_{2}$ Geometry 12

\section{Introduction}

There is by now a great deal of evidence that the correspondence between type IIB string theory on $\mathrm{AdS}_{5} \times \mathrm{S}^{5}$ and $\mathcal{N}=4$ super-Yang-Mills theory in four dimensions [1] extends to a more general duality between any gravitational theory on anti-de Sitter spaces and conformal field theories residing on the boundary of AdS [2]. Such a duality represents an explicit realization of the holographic principle [3]. In three dimensions, pure Einstein-AdS gravity was known to be related to a two-dimensional conformal field theory [4] even before the advent of the AdS/CFT correspondence. This is based on the fact that pure Einstein gravity in three dimensions can be written as a Chern-Simons theory [5], which is known to reduce to a WZNW model in presence of a boundary [6]. The boundary conditions for asymptotically AdS spaces [7] provide then the constraints for a Hamiltonian reduction from the WZNW model to Liouville field theory [4]. Recently the equivalence of pure gravity in three dimensions and two-dimensional Liouville theory has been extended in [8] to the case 
of positive cosmological constant, providing thus an explicit example of the proposed correspondence between de Sitter gravity and Euclidean conformal field theories [9].

Much less is known for two-dimensional anti-de Sitter gravity [10], which, in the spirit of AdS/CFT, should have a dual description in terms of a conformal quantum mechanical system [11 $]^{1}$. Progress in this direction has been made in [13], where it was shown that two-dimensional Jackiw-Teitelboim-AdS gravity [14] induces on the spacetime boundary a conformally invariant dynamics that can be described in terms of a de Alfaro-Fubini-Furlan model [15] coupled to an external source.

In the present paper we will derive a correspondence between two-dimensional AdS or dS gravity and a quantum mechanical system in an alternative way, using the fact that the Jackiw-Teitelboim (JT) model can be formulated as a topological gauge theory $[16,17]$. It will turn out that, in the same way in which a Chern-Simons theory in three dimensions reduces to a WZNW model in presence of a boundary, this $2 \mathrm{~d}$ topological gauge theory reduces to a nonlinear $\sigma$ model on a one-dimensional boundary.

The remainder of this paper is organized as follows: In section 2, the formulation of the JT model of $2 \mathrm{~d}$ dilaton gravity as a topological gauge theory is briefly reviewed. In section 3, we translate the boundary conditions for asymptotically $\mathrm{AdS}_{2}$ spaces $[18,19]$ into conditions for the fields appearing in the gauge theory. In the following section, it is shown that one must add boundary terms to the bulk action in order to have a well-defined variational principle. Since the bulk action is topological, the dynamics of the system is entirely encoded in these boundary terms, which give rise to a nonlinear $\sigma$ model in $0+1$ dimensions. In section 5 we perform a further reduction of the $\sigma$ model to a generalized two-particle Calogero-Sutherland model. This is similar to the familiar Hamiltonian reduction from the WZNW model to Liouville theory. Finally, in section 6, we generalize the considerations of sections 35, which refer to AdS gravity, to the case of positive cosmological constant.

\section{Two-dimensional (anti-)de Sitter gravity as a topological gauge theory}

It is well-known that the Jackiw-Teitelboim (JT) model [14] of dilaton gravity in two dimensions, with action given by

$$
S=\frac{1}{2} \int d^{2} x \sqrt{-g} \eta(R-2 \Lambda)
$$

where $\eta$ denotes the dilaton and $\Lambda$ the cosmological constant, can be formulated as an $\mathrm{SU}(1,1)$ topological gauge theory $[16,17]$. This is similar to the threedimensional case, where pure Einstein gravity can be written as a Chern-Simons

\footnotetext{
${ }^{1}$ Cf. also [12] and references therein.
} 
theory [5]. Apart from the gauge field, the gauge theory contains also a scalar field $\Phi$ in the adjoint of $\mathrm{SU}(1,1)$. The action reads ${ }^{2}$

$$
S=-\int \operatorname{Tr}(\Phi F)
$$

where $\Phi=\Phi^{A} \tau_{A}$ and $F=\frac{1}{2} F_{\mu \nu}^{A} \tau_{A} d x^{\mu} \wedge d x^{\nu}$ take values in the Lie algebra $\operatorname{su}(1,1)$. (The generators $\tau_{A}$ are given in the appendix). The field strength is given by

$$
F_{\mu \nu}=\partial_{\mu} A_{\nu}-\partial_{\nu} A_{\mu}+\left[A_{\mu}, A_{\nu}\right]
$$

The action (2.2) is invariant under the gauge transformations

$$
A_{\mu} \rightarrow g^{-1} A_{\mu} g+g^{-1} \partial_{\mu} g, \quad \Phi \rightarrow g^{-1} \Phi g .
$$

The equations of motion following from (2.2) read

$$
\begin{aligned}
F_{\mu \nu} & =0 \\
D_{\mu} \Phi & \equiv \partial_{\mu} \Phi+\left[A_{\mu}, \Phi\right]=0,
\end{aligned}
$$

so that the solutions are those of flat $\mathrm{SU}(1,1)$ connections in two dimensions, i. e. , locally one can write $A_{\mu}=g^{-1} \partial_{\mu} g$, with $g \in \mathrm{SU}(1,1)$.

To make contact with gravity, one decomposes the $\mathrm{su}(1,1)$ valued scalar and gauge fields according to

$$
\Phi^{A}=\left(\phi^{a}, \eta\right), \quad A_{\mu}^{A}=\left(\lambda e_{\mu}^{a}, \omega_{\mu}\right),
$$

where $e_{\mu}^{a}(a=0,1)$ and $\omega_{\mu}=\omega_{01 \mu}$ denote the zweibein and spin connection respectively. $\lambda$ is related to the cosmological constant by $\Lambda=\mp \lambda^{2}$, with the minus sign leading to AdS and the plus sign to dS gravity. The field strength $F_{\mu \nu}^{A}$ is written accordingly as

$$
F_{\mu \nu}^{A}=\left(\lambda T_{\mu \nu}^{a}, \mathcal{R}_{\mu \nu}\right)
$$

where $T_{\mu \nu}^{a}$ will correspond to the torsion tensor, and $\mathcal{R}_{\mu \nu}$ to the curvature tensor $[16,17]$. Vanishing torsion is enforced by the Lagrange multipliers $\phi^{a}$. The equivalence (at the classical level ${ }^{3}$ ) of (2.1) and (2.2) has been shown in $[16,17]$, so we refer to these papers for the details.

\footnotetext{
${ }^{2}$ Note that (2.2) can also be obtained by dimensional reduction of the $3 \mathrm{~d}$ Chern-Simons action.

${ }^{3}$ For a discussion of the quantum equivalence cf. [20], where it was shown that the theories are indeed equivalent in the light-cone gauge. A further analysis can be found in [21].
} 


\section{Asymptotic behaviour of the fields}

We will now focus on the case of negative cosmological constant. Due to Birkhoff's theorem of two-dimensional dilaton gravity, the general solution to the equations of motion following from (2.1) can be written in the form [22,23]

$$
\begin{aligned}
d s^{2} & =-\left(\lambda^{2} r^{2}-a^{2}\right) d t^{2}+\left(\lambda^{2} r^{2}-a^{2}\right)^{-1} d r^{2}, \\
\eta & =\eta_{0} \lambda r
\end{aligned}
$$

where $a$ and $\eta_{0}$ denote integration constants. Note that due to the presence of the dilaton, which represents a position-dependent coupling constant, the solutions with different sign of $a^{2}$ are physically inequivalent [23]. In particular, the solution with positive $a^{2}$ represents a black hole with event horizon at $r=a / \lambda$.

An asymptotically $\mathrm{AdS}_{2}$ geometry $[18,19]$ is one for which the metric behaves for $r \rightarrow \infty$ as

$$
g_{t t}=-\lambda^{2} r^{2}+\mathcal{O}(1), \quad g_{t r}=\mathcal{O}\left(\frac{1}{r^{3}}\right), \quad g_{r r}=\frac{1}{\lambda^{2} r^{2}}+\mathcal{O}\left(\frac{1}{r^{4}}\right) .
$$

The boundary conditions (3.2) must be completed by giving the asymptotic behaviour of the dilaton. As discussed in detail in [19,24], the appropriate boundary condition is

$$
\eta=\mathcal{O}(r),
$$

with a subleading term of $\mathcal{O}\left(r^{-1}\right)$ [24]. When translated in terms of the fields $\Phi, A$, the boundary conditions (3.2), (3.3) read

$$
\begin{aligned}
\Phi & =-\left[\eta_{0} \lambda r(1+\alpha(t))+\frac{2 a(t)}{r}\right] \tau_{0}+\left[\eta_{0} \lambda r(1+\beta(t))+\frac{2 b(t)}{r}\right] \tau_{2}+2 \gamma(t) \tau_{1}(3.4 \\
A_{t} & =\left[\lambda^{2} r+\frac{c(t)}{r}\right] \tau_{0}-\left[\lambda^{2} r+\frac{d(t)}{r}\right] \tau_{2}+\mathcal{O}\left(r^{-2}\right) \tau_{1} \\
A_{r} & =\left[r^{-1}+\mathcal{O}\left(r^{-3}\right)\right] \tau_{1}+\mathcal{O}\left(r^{-4}\right) \tau_{0}+\mathcal{O}\left(r^{-4}\right) \tau_{2}
\end{aligned}
$$

where the functions $\alpha(t), \beta(t), \gamma(t), a(t), b(t), c(t), d(t)$, that depend only on the boundary coordinate $t$, parametrize the subleading behaviour ${ }^{4}$.

Now some comments are in order. First of all, the asymptotics of the fields $\phi^{0}, \phi^{1}$ do not follow from (3.2) and (3.3), but rather can be inferred from the scalar equations

\footnotetext{
${ }^{4}$ The next to leading terms of $A_{r}$ were not parametrized, as they will not be used explicitely in what follows.
} 
of motion (2.6). Second, although one could consider general boundary conditions allowing for $\alpha(t) \neq \beta(t)$, we will define a configuration to be asymptotically $\mathrm{AdS}_{2}$ if $\alpha(t)=\beta(t)$. This definition is entirely consistent.

A well-defined asymptotics for the fields $\Phi$ and $A$ can be obtained after performing a gauge transformation (2.4), with group element $g$ given by

$$
g=\left(\begin{array}{c}
\cosh \theta \sinh \theta \\
\sinh \theta \cosh \theta
\end{array}\right), \quad \cosh \theta=\frac{1}{2}\left(\sqrt{\frac{f}{r}}+\sqrt{\frac{r}{f}}\right),
$$

where $f=f(t)$ denotes a function of $t$ to be specified later. The transformed fields $\Phi^{\prime}=g^{-1} \Phi g$ and $A^{\prime}=g^{-1} A g+g^{-1} d g$ obey, to leading order, the boundary conditions

$$
\begin{aligned}
& \Phi^{\prime}=-\left[\eta_{0} \lambda(1+\alpha) f+\frac{a-b}{f}\right] \tau_{0}+\left[\eta_{0} \lambda(1+\alpha) f-\frac{a-b}{f}\right] \tau_{2}+2 \gamma \tau_{1}, \\
& A_{t}^{\prime}=\left[\lambda^{2} f+\frac{c-d}{2 f}\right] \tau_{0}-\left[\lambda^{2} f-\frac{c-d}{2 f}\right] \tau_{2}+\frac{\dot{f}}{f} \tau_{1}, \\
& A_{r}^{\prime}=0 .
\end{aligned}
$$

For reasons that will become clear in the next section, we would like to have the boundary condition

$$
\Phi=\kappa(t) A_{t}
$$

with $\kappa(t)$ some function. (3.9) can be obtained from (3.8) by a second gauge transformation, with group element

$$
h=\left(\begin{array}{cc}
\exp (i \varphi(t)) & 0 \\
0 & \exp (-i \varphi(t))
\end{array}\right) .
$$

The transformed fields $\Phi^{\prime \prime}=h^{-1} \Phi^{\prime} h$ and $A^{\prime \prime}=h^{-1} A^{\prime} h+h^{-1} d h$ will then obey the boundary condition (3.9) if we choose the functions $f(t)$ and $\varphi(t)$ appearing in the transformation matrices $g$ and $h$ as solutions of the differential equations

$$
\begin{aligned}
& \dot{f}=-2 \gamma f \frac{2 \lambda^{2} f^{2}-(c-d)}{f^{2} \eta_{0} \lambda(1+\alpha)-(a-b)}, \\
& \dot{\varphi}=f \frac{2 \lambda^{2}(a-b)-\eta_{0} \lambda(1+\alpha)(c-d)}{f^{2} \eta_{0} \lambda(1+\alpha)-(a-b)} .
\end{aligned}
$$

The function $\kappa(t)$ appearing in (3.9) is then given by 


$$
\kappa(t)=\frac{4 f \gamma}{\dot{f}} .
$$

Note that the functions $f(t)$ and $\varphi(t)$ as well as the proportionality "constant" $\kappa(t)$ depend on the boundary fields $\alpha(t), \gamma(t), a(t), b(t), c(t)$ and $d(t)$. In what follows, we will drop the primes on $\Phi^{\prime \prime}$ and $A^{\prime \prime}$.

\section{Boundary terms in the action}

In the variation of the action (2.2) one encounters a term

$$
\int d^{2} x \operatorname{Tr}\left(\Phi \partial_{r} \delta A_{t}\right)=-\int d^{2} x \operatorname{Tr}\left(\partial_{r} \Phi \delta A_{t}\right)+\int d t \operatorname{Tr}\left(\Phi \delta A_{t}\right)
$$

This means that the action is not an extremum on-shell, instead $\delta S$ equals the surface term ${ }^{5} \int d t \operatorname{Tr}\left(\kappa(t) A_{t} \delta A_{t}\right)$. In order to cancel this, we must add a boundary term to the action (2.2), leading to the improved action

$$
S=\int d^{2} x \operatorname{Tr}\left(\Phi F_{r t}\right)-\frac{1}{2} \int d t \kappa(t) \operatorname{Tr}\left[\left(A_{t}\right)^{2}\right]
$$

The function $-\kappa(t) / 2$ can be absorbed into the time coordinate by defining $d \tau=-2 d t / \kappa(t)$

We recognize the $\Phi^{A}$ as Lagrange multipliers implementing the Gauss law constraint $F_{r \tau}^{A}=0$. In the functional integral one can integrate over $\Phi$ to obtain $\delta\left(F_{r \tau}\right)$,

$$
\int D A D \Phi e^{i S} \ldots=\int D A \delta\left(F_{r \tau}\right) e^{i \int d \tau \operatorname{Tr}\left[\left(A_{\tau}\right)^{2}\right]} \ldots
$$

The constraint $F_{r \tau}=0$ is easily solved by $A=g^{-1} d g^{6}$. Like in [6], we now change variables from $A$ to $g$ in the path integral,

$$
\int D A \delta\left(F_{r \tau}\right) e^{i \int d \tau \operatorname{Tr}\left[\left(A_{\tau}\right)^{2}\right]} \ldots=\int D g e^{i \int d \tau \operatorname{Tr}\left[\left(g^{-1} \partial_{\tau} g\right)^{2}\right]} \ldots
$$

We have thus derived an effective action

$$
S_{\mathrm{eff}}=\int d \tau \operatorname{Tr}\left[\left(g^{-1} \partial_{\tau} g\right)^{2}\right]
$$

\footnotetext{
${ }^{5}$ Here we used the boundary condition (3.9).

${ }^{6}$ As we consider the universal covering space of $\mathrm{AdS}_{2}$, holonomies can be dropped.
} 
for the problem under consideration. (4.5) describes a nonlinear $\sigma$ model in $0+1$ dimensions.

If we write $g \in \mathrm{SU}(1,1)$ in the Iwasawa decomposition

$$
g=\left(\begin{array}{cc}
e^{i u / 2} & 0 \\
0 & e^{-i u / 2}
\end{array}\right)\left(\begin{array}{c}
\cosh \frac{\vartheta}{2} \sinh \frac{\vartheta}{2} \\
\sinh \frac{\vartheta}{2} \cosh \frac{\vartheta}{2}
\end{array}\right)\left(\begin{array}{cc}
e^{i v / 2} & 0 \\
0 & e^{-i v / 2}
\end{array}\right)
$$

the action (4.5) reads

$$
S_{\mathrm{eff}}=\frac{1}{2} \int d \tau\left(\dot{\vartheta}^{2}-\dot{u}^{2}-\dot{v}^{2}-2 \dot{u} \dot{v} \cosh \vartheta\right)
$$

where a dot denotes differentiation with respect to $\tau$. Defining $x^{1}=u, x^{2}=v$, $x^{3}=\vartheta$, one can write

$$
S_{\mathrm{eff}}=\frac{1}{2} \int g_{i j} \dot{x}^{i} \dot{x}^{j} d \tau
$$

where $i, j=1,2,3$ and

$$
\left(g_{i j}\right)=\left(\begin{array}{ccc}
-1 & -\cosh \vartheta & 0 \\
-\cosh \vartheta & -1 & 0 \\
0 & 0 & 1
\end{array}\right)
$$

is a constant curvature metric on the group manifold $\mathrm{SU}(1,1)$. (4.9) admits the six Killing vectors

$$
\begin{array}{ll}
L_{0}=-i \partial_{v}, & L_{ \pm}=e^{\mp i v}\left[i \partial_{\theta} \pm \operatorname{coth} \vartheta \partial_{v} \mp \operatorname{cosech} \vartheta \partial_{u}\right], \\
\tilde{L}_{0}=-i \partial_{u}, & \tilde{L}_{ \pm}=e^{\mp i u}\left[i \partial_{\theta} \pm \operatorname{coth} \vartheta \partial_{u} \mp \operatorname{cosech} \vartheta \partial_{v}\right],
\end{array}
$$

which satisfy the commutation relations

$$
\begin{array}{ll}
{\left[L_{ \pm}, L_{0}\right]= \pm L_{ \pm},} & {\left[L_{+}, L_{-}\right]=2 L_{0}} \\
{\left[\tilde{L}_{ \pm}, \tilde{L}_{0}\right]= \pm \tilde{L}_{ \pm},} & {\left[\tilde{L}_{+}, \tilde{L}_{-}\right]=2 \tilde{L}_{0}}
\end{array}
$$

and $[L, \tilde{L}]=0$. The Killing vectors generate thus the algebra $\operatorname{su}(1,1) \times \operatorname{su}(1,1)$, corresponding to the invariance of the action (4.5) under $g \rightarrow h g \tilde{h}$, with $h, \tilde{h} \in$ $\mathrm{SU}(1,1)$ independent of $\tau$. 


\section{Further reduction}

In this section we will show that, due to the presence of cyclic coordinates, the $\sigma$ model (4.5) can be further reduced to a simple quantum mechanical system.

As the Lagrangian in (4.7) does not depend on $u, v$, the conjugate momenta

$$
\pi_{u}=-\dot{u}-\dot{v} \cosh \vartheta, \quad \pi_{v}=-\dot{v}-\dot{u} \cosh \vartheta
$$

are constants of motion. In order to implement the constraints $\pi_{u, v}=$ const, one has to go from (4.7) to the reduced action (Routhian function)

$$
S_{\mathrm{eff}} \rightarrow S_{\mathrm{eff}}-\int d \tau\left[\dot{u} \pi_{u}+\dot{v} \pi_{v}\right]
$$

i. e., one has to perform a partial Legendre transformation with respect to $\dot{u}, \dot{v}$. In this way, one finally obtains

$$
S_{\text {eff }}=\frac{1}{2} \int d \tau\left[\dot{\vartheta}^{2}-\frac{\left(\pi_{u}-\pi_{v}\right)^{2}}{4 \sinh ^{2} \frac{\vartheta}{2}}+\frac{\left(\pi_{u}+\pi_{v}\right)^{2}}{4 \cosh ^{2} \frac{\vartheta}{2}}\right],
$$

which is a generalization of the two-particle Calogero-Sutherland model [25]. The relationship between $\mathrm{AdS}_{2}$ gravity and the Calogero model was first pointed out in $[26]$.

Note that the only generators of the conformal algebra (4.10) that commute with $\pi_{v}=L_{0}$ and $\pi_{u}=\tilde{L}_{0}$ are $L_{0}$ and $\tilde{L}_{0}$, which means that the conformal symmetry is broken by the reduction to (5.3). Conformal transformations actually relate different $\pi_{u, v}$, and therefore fixing these momenta breaks the invariance under $\mathrm{su}(1,1) \times$ $\operatorname{su}(1,1)$.

\section{Positive cosmological constant}

In the case of positive cosmological constant $\Lambda=\lambda^{2}$, the general solution to the equations of motion following from (2.1) can be written as

$$
\begin{aligned}
d s^{2} & =-\left(a^{2}-\lambda^{2} r^{2}\right) d t^{2}+\left(a^{2}-\lambda^{2} r^{2}\right)^{-1} d r^{2}, \\
\eta & =\eta_{0} \lambda r .
\end{aligned}
$$

For a more detailed discussion of the geometry of two-dimensional de Sitter space we refer to the appendix. Note in particular that for $r>a / \lambda, r$ becomes a timelike coordinate $^{7}$, so that the dilaton is now time-dependent outside the static patch.

\footnotetext{
${ }^{7}$ Of course $\partial_{r}$ is always timelike for $a^{2} \leq 0$.
} 
In what follows, we will be particularly interested in the behaviour of the gravitational fields near the past boundary $\mathcal{I}^{-}$at $r \rightarrow \infty$. The boundary conditions for an asymptotically $\mathrm{dS}_{2}$ geometry can be found by analytically continuing (3.2) and (3.3). This yields

$$
g_{t t}=\lambda^{2} r^{2}+\mathcal{O}(1), \quad g_{t r}=\mathcal{O}\left(\frac{1}{r^{3}}\right), \quad g_{r r}=-\frac{1}{\lambda^{2} r^{2}}+\mathcal{O}\left(\frac{1}{r^{4}}\right)
$$

and $\eta=\mathcal{O}(r)$. Alternatively, one can obtain these boundary conditions by requiring the subleading terms to fall off fast enough to ensure that conserved charges are well-defined, but slow enough to make the theory non-trivial. This singles out the behaviour (6.2).

At this point, one can proceed analogously to the AdS case, and translate (6.2) into boundary conditions for the gauge field $A$ and the scalar $\Phi$. Again, these conditions turn out to be gauge equivalent to $\Phi=\kappa(t) A_{t}, A_{r}=0$, with some function $\kappa(t)$, as in section 3. Taking into account the boundary terms ${ }^{8}$ necessary to have a well-defined variational principle, integrating over the Lagrange multiplier $\Phi$ in the path integral, and changing variables from $A=g^{-1} d g$ to $g$, one derives also in the dS case an effective $\sigma$ model action (4.5), which allows a further reduction to the quantum mechanics $(5.3)^{9}$. Note that these one-dimensional models live now in Euclidean space, since the boundaries of $\mathrm{dS}_{2}$ are spacelike.

\section{Conclusions}

To sum up, we showed that the asymptotic dynamics of two-dimensional de Sitter or anti-de Sitter Jackiw-Teitelboim gravity is encoded in a generalized two-particle Calogero-Sutherland quantum mechanical model. We started by formulating the JT model of (A)dS gravity in two dimensions as a topological gauge theory, which, by virtue of the $(\mathrm{A}) \mathrm{dS}$ boundary conditions, reduces to a nonlinear sigma model on the one-dimensional boundary of (A)dS space. The appearance of cyclic coordinates allows then a further reduction to the quantum mechanical system (5.3). We have thus generalized the known results in three dimensions, where the asymptotic dynamics

\footnotetext{
${ }^{8}$ Note that $\mathrm{dS}_{2}$ has two boundaries, the past boundary $\mathcal{I}^{-}$and the future boundary $\mathcal{I}^{+}$, so in principle one has to consider two boundary terms, which should lead to a quantum mechanical system living on a disconnected manifold. However, since we are only interested in the asymptotic dynamics of the gravitational fields near $\mathcal{I}^{-}$, we ignore surface terms arising at $\mathcal{I}^{+}$. Of course the same problem appears in the $\mathrm{AdS}_{2}$ case, since two-dimensional anti-de Sitter space, unlike its higher-dimensional cousins, has two boundaries. A possible way to eliminate the second boundary is to cut the spacetime at $r=0$, in order that the position-dependent coupling constant represented by the dilaton is positive [19].

${ }^{9} \mathrm{~A}$ similar reduction to a quantum mechanical system was performed in [27]. There the boundary was the horizon, whereas we consider the boundaries at infinity.
} 
of pure Einstein gravity with negative [4] or positive [8] cosmological constant is described by Liouville field theory, obtained as the Hamiltonian reduction of a WZNW model.

Note that the JT model receives no local quantum corrections at all orders of perturbation theory [28]. Probably, this means that our results can be extended far beyond the approximation they were actually derived.

It would also be interesting to consider the supersymmetric generalization of the topological gauge theory (2.2) [17], which is (at least classically) equivalent to an (A)dS supergravity model in two dimensions. These two-dimensional supergravity theories should then have an equivalent description in terms of a superextension of the quantum mechanical system (5.3).

As also the CGHS model [29] of two-dimensional dilaton gravity can be formulated as a topological gauge theory based on the centrally extended Poincaré group [30], an analogous reduction to a one-dimensional quantum mechanics should be possible. The field equations following from the CGHS model in the form

$$
S=\frac{1}{2} \int d^{2} x \sqrt{-g}(\eta R-\Lambda)
$$

imply $R=0$, so the boundary conditions are now those of asymptotically flat metrics. One thus expects the quantum mechanical model to be defined on a lightlike line. It would be tempting to see explicitely what this model is.

These issues are currently under investigation.

\section{Acknowledgments}

This work was partially supported by INFN, MURST and by the European Commission RTN program HPRN-CT-2000-00131, in which S. C. , D. K. and D. Z. are associated to the University of Torino. The authors would like to thank R. Jackiw, T. Strobl and D. Vassilevich for useful comments. 


\section{A. Conventions}

\section{A.1 Anti-de Sitter}

An element $M$ of the Lie algebra $\mathrm{su}(1,1)$ satisfies

$$
M_{b}^{a}=-\eta_{b c} \bar{M}_{d}^{c} \eta^{d a}, \quad \operatorname{Tr} M=0
$$

where a bar denotes complex conjugation, and $\left(\eta_{a b}\right)=\operatorname{diag}(-1,1)$. We choose as $\mathrm{su}(1,1)$ generators

$$
\tau_{0}=\frac{1}{2}\left(\begin{array}{cc}
i & 0 \\
0 & -i
\end{array}\right), \quad \tau_{1}=\frac{1}{2}\left(\begin{array}{ll}
0 & 1 \\
1 & 0
\end{array}\right), \quad \tau_{2}=\frac{1}{2}\left(\begin{array}{cc}
0 & -i \\
i & 0
\end{array}\right) .
$$

They are normalized according to

$$
\operatorname{Tr}\left(\tau_{A} \tau_{B}\right)=\frac{1}{2} \eta_{A B}
$$

where $\left(\eta_{A B}\right)=\operatorname{diag}(-1,1,1)$ is the inner product on the Lie algebra. The generators (A.2) satisfy the relation (A.1), and $\left[\tau_{A}, \tau_{B}\right]=-\epsilon_{A B C} \tau^{C}$, with $\epsilon_{012}=1$.

Finally, $d t \wedge d r$ is chosen to have positive orientation.

\section{A.2 De Sitter}

In order to get a positive cosmological constant we interchange $\tau_{0}$ and $\tau_{1}$ in (A.2), i. e., we take

$$
\tau_{0}=\frac{1}{2}\left(\begin{array}{ll}
0 & 1 \\
1 & 0
\end{array}\right), \quad \tau_{1}=\frac{1}{2}\left(\begin{array}{cc}
i & 0 \\
0 & -i
\end{array}\right), \quad \tau_{2}=\frac{1}{2}\left(\begin{array}{cc}
0 & -i \\
i & 0
\end{array}\right)
$$

with the normalization

$$
\operatorname{Tr}\left(\tau_{A} \tau_{B}\right)=-\frac{1}{2} \eta_{A B}
$$

where now $\left(\eta_{A B}\right)=\operatorname{diag}(-1,1,-1)$. All the other conventions are as in subsection A.1. 


\section{B. $\mathrm{dS}_{2}$ Geometry}

Consider $\mathbb{R}_{1}^{2}$ with coordinates $X^{0}, X^{1}, X^{2}$ and metric $(-1,1,1)$. Two-dimensional de Sitter space can then be defined as the hypersurface

$$
-\left(X^{0}\right)^{2}+\left(X^{1}\right)^{2}+\left(X^{2}\right)^{2}=\lambda^{-2}
$$

Fix now

$$
\left(X^{0}\right)^{2}-\left(X^{1}\right)^{2}=\frac{r^{2}}{a^{2}}-\lambda^{-2}
$$

so that $\left(X^{2}\right)^{2}=r^{2} / a^{2}$, and parametrize the hyperbolae of fixed $r$ by

$$
X^{0}=\frac{1}{\lambda a} \sqrt{\lambda^{2} r^{2}-a^{2}} \cosh a \lambda t, \quad X^{1}=\frac{1}{\lambda a} \sqrt{\lambda^{2} r^{2}-a^{2}} \sinh a \lambda t .
$$

This leads to the induced metric

$$
d s^{2}=-\left(a^{2}-\lambda^{2} r^{2}\right) d t^{2}+\left(a^{2}-\lambda^{2} r^{2}\right)^{-1} d r^{2} .
$$

Note that the coordinate $t$ is noncompact.

Alternatively, we can fix

$$
\left(X^{1}\right)^{2}+\left(X^{2}\right)^{2}=\frac{r^{2}}{a^{2}}+\lambda^{-2}
$$

so that $\left(X^{0}\right)^{2}=r^{2} / a^{2}$, and parametrize the circles of fixed $r$ by

$$
X^{1}=\frac{1}{\lambda a} \sqrt{\lambda^{2} r^{2}+a^{2}} \cos a \lambda t, \quad X^{2}=\frac{1}{\lambda a} \sqrt{\lambda^{2} r^{2}+a^{2}} \sin a \lambda t .
$$

The induced metric on the hypersurface $(\mathbb{B} .1)$ is now

$$
d s^{2}=-\left(a^{2}+\lambda^{2} r^{2}\right) d t^{2}+\left(a^{2}+\lambda^{2} r^{2}\right)^{-1} d r^{2} .
$$

The coordinate $t$ is now compact, $t \sim t+2 \pi / \lambda a$. The boundaries at $r \rightarrow \pm \infty$ are thus circles. $t, r$ in $(\underline{B .7})$ are global coordinates. Defining

$$
r=-\frac{a}{\lambda} \sinh \tau, \quad t=\frac{1}{\lambda a} \phi
$$


where $\phi$ is identified modulo $2 \pi$, $(\underline{B .7})$ can be rewritten as

$$
\lambda^{2} d s^{2}=-d \tau^{2}+\cosh ^{2} \tau d \phi^{2}
$$

which describes $\mathrm{dS}_{2}$ as a contracting/expanding one-sphere. $\tau \rightarrow-\infty$ corresponds to the past boundary $\mathcal{I}^{-}$, whereas $\tau \rightarrow \infty$ corresponds to the future boundary $\mathcal{I}^{+}$. 


\section{References}

[1] J. Maldacena, "The large $N$ limit of superconformal field theories and supergravity," Adv. Theor. Math. Phys. 2 (1998) 231 [Int. J. Theor. Phys. 38 (1998) 1113] arXiv:hepth/9711200.

[2] E. Witten, "Anti-de Sitter space and holography," Adv. Theor. Math. Phys. 2 (1998) 253 arXiv:hep-th/9802150.

[3] G. 't Hooft, "Dimensional Reduction In Quantum Gravity," arXiv:gr-qc/9310026;

L. Susskind, "The World as a hologram," J. Math. Phys. 36 (1995) 6377 arXiv:hepth/9409089.

[4] O. Coussaert, M. Henneaux and P. van Driel, "The Asymptotic dynamics of threedimensional Einstein gravity with a negative cosmological constant," Class. Quant. Grav. 12 (1995) 2961 arXiv:gr-qc/9506019.

[5] A. Achucarro and P. K. Townsend, "A Chern-Simons Action For Three-Dimensional Anti-De Sitter Supergravity Theories," Phys. Lett. B 180 (1986) 89;

E. Witten, "(2+1)-Dimensional Gravity As An Exactly Soluble System," Nucl. Phys. B 311 (1988) 46.

[6] S. Elitzur, G. W. Moore, A. Schwimmer and N. Seiberg, "Remarks On The Canonical Quantization Of The Chern-Simons-Witten Theory," Nucl. Phys. B 326 (1989) 108.

[7] J. D. Brown and M. Henneaux, "Central Charges In The Canonical Realization Of Asymptotic Symmetries: An Example From Three-Dimensional Gravity," Commun. Math. Phys. 104 (1986) 207.

[8] S. Cacciatori and D. Klemm, "The asymptotic dynamics of de Sitter gravity in three dimensions," Class. Quant. Grav. 19 (2002) 579 arXiv:hep-th/0110031.

[9] A. Strominger, "The dS/CFT correspondence," JHEP 0110 (2001) 034 arXiv:hepth/0106113.

[10] A. Strominger, "AdS ${ }_{2}$ quantum gravity and string theory," JHEP 9901 (1999) 007 arXiv:hep-th/9809027.

[11] P. Claus, M. Derix, R. Kallosh, J. Kumar, P. K. Townsend and A. Van Proeyen, "Black holes and superconformal mechanics," Phys. Rev. Lett. 81 (1998) 4553 arXiv:hepth/9804177.

[12] S. Cacciatori, D. Klemm and D. Zanon, " $w_{\infty}$ algebras, conformal mechanics, and black holes," Class. Quant. Grav. 17 (2000) 1731 arXiv:hep-th/9910065.

[13] M. Cadoni, P. Carta, D. Klemm and S. Mignemi, "AdS ${ }_{2}$ gravity as conformally invariant mechanical system," Phys. Rev. D 63 (2001) 125021 arXiv:hep-th/0009185. 
[14] C. Teitelboim, "The Hamiltonian structure of two-dimensional space-time and its relation with the conformal anomaly," in Quantum Theory of Gravity, edited by S. M. Christensen (Hilger, Bristol); R. Jackiw, "Liouville field theory: a twodimensional model for gravity?," ibid.

[15] V. de Alfaro, S. Fubini and G. Furlan, "Conformal Invariance In Quantum Mechanics," Nuovo Cim. A 34 (1976) 569.

[16] K. Isler and C. A. Trugenberger, "A Gauge Theory Of Two-Dimensional Quantum Gravity," Phys. Rev. Lett. 63 (1989) 834.

[17] A. H. Chamseddine and D. Wyler, "Topological Gravity In (1+1)-Dimensions," Nucl. Phys. B 340 (1990) 595.

[18] M. Cadoni and S. Mignemi, "Entropy of 2D black holes from counting microstates," Phys. Rev. D 59 (1999) 081501 arXiv:hep-th/9810251.

[19] M. Cadoni and S. Mignemi, "Asymptotic symmetries of $\mathrm{AdS}_{2}$ and conformal group in d = 1," Nucl. Phys. B 557 (1999) 165 arXiv:hep-th/9902040.

[20] H. Terao, "Quantum analysis of Jackiw and Teitelboim's model for (1+1)-D gravity and topological gauge theory," Nucl. Phys. B 395 (1993) 623 arXiv:hep-th/9205030.

[21] P. Schaller and T. Strobl, "Diffeomorphisms versus nonabelian gauge transformations: An Example of (1+1)-dimensional gravity," Phys. Lett. B 337 (1994) 266 arXiv:hepth/9401110.

[22] D. Christensen and R. B. Mann, "The Causal structure of two-dimensional spacetimes," Class. Quant. Grav. 9 (1992) 1769 arXiv:hep-th/9203050;

A. Achucarro and M. E. Ortiz, "Relating black holes in two-dimensions and threedimensions," Phys. Rev. D 48 (1993) 3600 arXiv:hep-th/9304068;

J. P. Lemos and P. M. Sa, "Nonsingular constant curvature two-dimensional black hole," Mod. Phys. Lett. A 9 (1994) 771 arXiv:gr-qc/9309023; "The Black Holes Of A General Two-Dimensional Dilaton Gravity Theory," Phys. Rev. D 49 (1994) 2897 [Erratum-ibid. D 51 (1994) 5967] arXiv:gr-qc/9311008.

[23] M. Cadoni and S. Mignemi, "Nonsingular four-dimensional black holes and the JackiwTeitelboim theory," Phys. Rev. D 51 (1995) 4319 arXiv:hep-th/9410041.

[24] M. Cadoni and S. Mignemi, "Symmetry breaking, central charges and the $\mathrm{AdS}_{2} / \mathrm{CFT}_{1}$ correspondence," Phys. Lett. B 490 (2000) 131 arXiv:hep-th/0002256.

[25] B. Sutherland, "Exact Results For A Quantum Many Body Problem In OneDimension," Phys. Rev. A 4 (1971) 2019.

[26] G. W. Gibbons and P. K. Townsend, "Black holes and Calogero models," Phys. Lett. B 454 (1999) 187 arXiv:hep-th/9812034. 
[27] J. Gegenberg, G. Kunstatter and T. Strobl, "Statistical mechanical entropy of twodimensional black holes," arXiv:gr-qc/9607055; "Edge states and entropy of 2d black holes," Phys. Rev. D 55 (1997) 7651 arXiv:gr-qc/9612033].

[28] W. Kummer, H. Liebl and D. V. Vassilevich, "Exact path integral quantization of generic 2-D dilaton gravity," Nucl. Phys. B 493 (1997) 491 arXiv:gr-qc/9612012.

[29] C. G. Callan, S. B. Giddings, J. A. Harvey and A. Strominger, "Evanescent Black Holes," Phys. Rev. D 45 (1992) 1005 arXiv:hep-th/9111056.

[30] D. Cangemi and R. Jackiw, "Gauge invariant formulations of lineal gravity," Phys. Rev. Lett. 69 (1992) 233 arXiv:hep-th/9203056]; "Quantum states of string inspired lineal gravity," Phys. Rev. D 50 (1994) 3913 [arXiv:hep-th/9403045]. 